This is the peer reviewed version of the following article: Price, P. and Lee, S. and Affandi, J. and Parsons, R. and Naylor, L. and Watts, G. and Irish, A. 2017. Cytomegalovirus antibody and vascular pathology in renal transplant recipients. Journal of Medical Virology. 89 (1): pp. 177-181, which has been published in final form at http://doi.org/10.1002/jmv.24602. This article may be used for noncommercial purposes in accordance with Wiley Terms and Conditions for SelfArchiving at http://olabout.wiley.com/WileyCDA/Section/id-828039.html 


\section{Cytomegalovirus antibody and vascular pathology in renal transplant recipients}

Patricia Price ${ }^{1,2,3}$, Silvia Lee ${ }^{1,2,4}$, Jacquita Affandi ${ }^{1,2}$, Richard Parsons ${ }^{5}$, Louise H. Naylor ${ }^{6}$, Gerald F. Watts ${ }^{3}$, Ashley Irish $^{3,7}$

${ }^{1}$ School of Biomedical Science, Curtin University, Bentley, Australia

${ }^{2}$ Curtin Health Innovation Research Institute, Curtin University, Bentley, Australia

${ }^{3}$ School of Medicine \& Pharmacology, University of Western Australia, Nedlands, Australia

${ }^{4}$ Department of Microbiology, Royal Perth Hospital, Perth, Australia

${ }^{5}$ School of Pharmacy, Curtin University, Bentley, Australia

${ }^{6}$ Sport Science, Exercise and Health, University of Western Australia, Nedlands, Australia

${ }^{7}$ Renal Unit, Fiona Stanley hospital, Murdoch, Australia

\section{Corresponding author:}

A/Professor Patricia Price

School of Biomedical Science, Curtin University of Technology, Bentley

Australia

Tel: +61 892669716

Email: patricia.price@,curtin.edu.au

\section{Key words:}

cytomegalovirus, cardiovascular disease, renal transplantation, aging 


\begin{abstract}
Background:

Cytomegalovirus (CMV) has been linked with vascular pathology and is a common infectious complication of renal transplantation. We addressed the relative importance of transplantation per se and CMV seropositivity as determinants of vascular pathology.
\end{abstract}

\title{
Findings:
}

Levels of CMV antibody increased with age and were higher in renal transplant recipients than healthy controls $(\mathrm{p}<0.001)$. The optimal general linear model predicting vascular endothelial function through flow mediated-dilation of the brachial artery $\left(F M D ; R^{2}=0.31, p<0.0001\right)$ included CMV seropositivity as a marginal effect $(p=0.068)$, with age $(p=0.013,<55$ years), gender $(p<0.0001)$ and transplantation $(p<0.0001)$. The same procedure identified no influence of CMV antibodies on maximum thickness of the carotid arterial wall, with the optimal model $\left(\mathrm{R}^{2}=0.30, \mathrm{p}<0.001\right)$ being based entirely on age $(\mathrm{p}<0.0001)$.

\section{Conclusions:}

The presence of CMV antibody was associated weakly with the endothelial dysfunction independent of age and transplantation. Validation of this association with other measures of CMV burden is clinically important because periodic CMV prophylaxis of selected patients stable on anti-rejection therapy is feasible and may reduce vascular pathology. 


\section{INTRODUCTION}

Most people carry cytomegalovirus (CMV) as a lifelong latent infection, with periodic reactivations triggered by inflammation or stress. CMV is also a common infectious complication of renal transplantation (1). Before prophylaxis was available, one in three renal transplant recipients (RTR) experienced pathologies associated with CMV. For example; 12 of 141 RTR in one study, died with disseminated CMV infection (2). In Australia, it is common practice to administer valganciclovir as prophylaxis for 12-26 weeks after transplantation, based on donor and recipient CMV seropositivity and clinical risk. Under a similar regimen, CMV replication was detected in $33 \%$ of previously seropositive RTR and $21 \%$ of those who were seronegative at transplantation (3). However the long-term use of valganciclovir after this initial prophylactic period is not standard as there are uncertain benefits and cost. However CMV reactivations are implicated in deterioration in renal function (4), new onset type II diabetes (5) and vascular pathologies. Cardiovascular disease after renal transplantation is a common long-term complication of successful renal transplantation and is more frequent in CMV-positive patients $(6,7)$. Periodic CMV prophylaxis of selected patients stable on anti-rejection therapy is feasible and has the potential to reduce these adverse outcomes.

Several studies associate the presence of CMV-DNA in tissues removed during surgery for abdominal aortic aneurysm with expression of inflammatory mediators $(8,9)$. Higher CMV antibody titres are linked with increased blood pressure in young men (10) and coronary artery disease requiring surgery (11). Here we assess whether levels of CMV antibodies or a simple dichotomy of seropositive versus negative independently predict large vessel health assessed by carotid intima media thickness (cIMT) and flow mediated dilatation (FMD) of the brachial artery. cIMT is an independent predictor of clinical cardiovascular events, including myocardial infarction and stroke (12). Brachial arterial FMD is assessed by high-resolution ultrasound after blood flow is occluded for a few minutes. The measurement reflects endothelium-dependent vasodilator function. It is validated as a surrogate of endothelial function of the coronary circulation, and associates with prevalent and incident cardiovascular disease (13). 


\section{METHODS}

\section{Patients and controls}

Renal transplant recipients (RTR; n=82) were recruited from the renal clinics at Royal Perth Hospital (RPH), Western Australia. Inclusion criteria were clinically stable $>2$ years after transplantation, no CMV disease or reactivation within 6 months of blood collection and no antiviral treatment. Age-matched healthy controls $(n=81)$ were recruited through local advertisements. Demographic details are provided in Table 1. Written informed consent was obtained from each participant. The project was approved by the Human Ethics Committees of Royal Perth Hospital, University of Western Australia and Curtin University.

\section{DNA and antibody}

CMV-reactive IgG was quantitated using a lysate of human foreskin fibroblasts (HFF) infected with strain AD169. Cells were harvested after 7 days, sonicated and stored at $-80^{\circ} \mathrm{C}$. Uninfected HFF were prepared as a negative control. Parallel microtitre plates were coated at $0.5 \mu \mathrm{g} / \mathrm{ml}$ (overnight, $4^{0} \mathrm{C}$ ) with $\mathrm{CMV}$ lysate, HFF lysate, CMV gB (glycoprotein B) prepared in hamster ovary cells (Chiron Diagnostics; Medfield, MA) and CMV IE-1 (Immediate early 1) prepared in E.coli (Miltenyi Biotech; Cologne, Germany). Plates were then blocked with 5\%BSA in PBS and washed. Plasma samples pre-diluted in 2\%BSA in PBS were run alongside standard plasma from a CMV-seropositive healthy donor and assigned a value of 1000 arbitrary units (AU/ml) for each antigen. Plates were washed after $2 \mathrm{hrs}$ and horse-radish peroxide conjugated anti-human IgG (Sigma-Aldrich; St Louis, MI) diluted 1:4000 in 2\%BSA in PBS was added for $1 \mathrm{hr}$. Tetramethylbenzidine substrate (Sigma-Aldrich) was added, colour development was stopped with $1 \mathrm{M} \mathrm{H}_{2} \mathrm{SO}_{4}$ and plates were read at $450 \mathrm{~nm}$. CMV seronegativity was defined using the CMV lysate assay as $<3$ standard deviations above the mean antibody levels recorded in 11 samples that were seronegative by the ARCHITECT CMV IgG assay (Abbott Diagnostic Systems; Lake Forrest, IL), creating a cut-off of $3623 \mathrm{AU} / \mathrm{ml}$. As a few individuals meeting this criterion had antibodies detectable with $\mathrm{gB}$ or IE-1, analyses were repeated using cut-offs of $340 \mathrm{AU} / \mathrm{ml} \mathrm{gB}$ and 3500AU/ml IE-1 antibody.

\section{Clinical assessments}

To assess cIMT, the common carotid arteries were examined in accordance with guidelines from the American Society of Echocardiography (14). Briefly; the anterolateral, posterolateral and mediolateral planes were acquired using a 12-15 MHz multi-frequency linear array probe attached to a high resolution ultrasound machine (Aspen, Acuson; Mountain View, CA). Subjects lay supine with a slight hyperextension of the neck and a $45^{\circ}$ lateral flexion away from the side 
scanned. The vessel was scanned to exclude any carotid plaque. An R-wave triggered optimal recording of the far wall, $1 \mathrm{~cm}$ proximal to the carotid bulb, was directly encoded and stored as a digital DICOM file for post hoc analysis.

After a 10 to 20-minute rest period, brachial artery diameter response to FMD was assessed using a 12-15 MHz multi-frequency linear array probe attached to a high resolution ultrasound machine, (15). Briefly, subjects lay supine with their arms extended at $\sim 80^{\circ}$ from their torso. A rapid inflation/deflation pneumatic cuff was placed around the arm distal to the olecranon process. When an optimal B-mode image was obtained, images were collected using a fixed insonation angle $\left(<60^{\circ}\right)$. Baseline images were recorded for $1 \mathrm{~min}$, before the forearm cuff was inflated to $220 \mathrm{~mm} \mathrm{Hg}$ for $5 \mathrm{mins}$. Recording resumed $30 \mathrm{sec}$ prior to cuff deflation, and continued for $5 \mathrm{mins}$ post-deflation. FMD is presented as the relative (\%) rise from the baseline diameter.

Diameters were analysed with custom-designed edge-detection and wall-tracking software to minimise investigator bias. Briefly; a region of interest is drawn around the B-mode image of the vessel segment, and the edges are detected automatically. Over 200 individual measurements are taken from each frame. For cIMT analysis, the median value is calculated to represent a single "composite cIMT". Three sets of these composite values from common carotid arteries are used to determine the mean lumen diameter and cIMT. Analyses of the reproducibility gave a CV of 6.7$10.5 \%$ for FMD (16) and 3.5\% for cIMT (17).

\section{Statistical analysis}

Statistical analyses were performed using Prism 5 (GraphPad Software, La Jolla, CA) and SAS (Version 9.2, 2008; Cary, NC). Univariate comparisons used non-parametric Mann-Whitney tests and Spearman's correlations. Multivariate associations between independent variables (transplantation, age and gender) and measures of CMV antibody (lysate, gB, IE-1) as dependent variables were sought with a separate general linear model (GLM) for each outcome. GLM were then developed with \%FMD or maximum cIMT as the outcome, to assess the influence of transplantation, gender, age (in quartiles), BMI (in tertiles), systolic and diastolic blood pressures (in quartiles) and CMV antibodies (in quartiles). Categories were created to assess whether the relationship with \%FMD approximated linearity. They were amalgamated where there appeared to be a 'step function' rather than a linear relationship, or removed if the relationship appeared to be linear. In each case, backward elimination was used to obtain the most parsimonious model. A $\mathrm{p}$-value $<0.05$ was taken to indicate a statistically significant association, but $\mathrm{p}<0.10$ was noted. 


\section{RESULTS AND DISCUSSION}

The 82 RTR cases and 81 healthy controls were similar in age and gender (Table 1). RTR had significantly higher levels of antibody reactive with CMV (lysate, $p<0.0001 ; g B, p<0.0001$; and IE-1, $\mathrm{p}=0.009$ ) than controls. This remained true when the analysis was restricted to CMV seropositive individuals (Table 1$)$. The optimal GLM for CMV lysate $\left(\mathrm{R}^{2}=0.19, \mathrm{p}<0.001\right)$ included age $(p=0.006)$ and transplantation $(p<0.0001)$ as significantly associated variables. Similar results were obtained with $\mathrm{gB}$ antibody $\left(\mathrm{R}^{2}=0.16, \mathrm{p}<0.0001\right.$ with age at $\mathrm{p}=0.027$ and transplantation at $\mathrm{p}<0.0001)$, but the optimal model for IE-1 antibody $\left(\mathrm{R}^{2}=0.12, \mathrm{p}<0.001\right)$ included transplantation $(p=0.007)$, gender $(p=0.004)$ and age $(p=0.025)$. In univariate analyses, gender affected levels of IE-1 antibodies (Wilcoxon rank sum, $\mathrm{p}=0.013$ ).

When RTR and controls were considered together, levels of CMV-reactive antibodies were inversely related to the \%FMD [lysate $\mathrm{r}=-0.27, \mathrm{p}=0.0008 ; \mathrm{gB} r=-0.29, \mathrm{p}=0.0003$; IE-1 $\mathrm{r}=-0.22$, $\mathrm{p}=0.0065]$ suggesting an association with impaired vascular function. While these negative associations persisted amongst RTR and control participants analysed separately, the correlations were not significantly different from zero (data not shown). This reflected the reduced range of CMV antibody and \%FMD levels, and smaller sample sizes. Accordingly amongst individuals who were CMV-seropositive, univariate analyses (Wilcoxon tests) showed that RTR had impaired vascular function when assessed by the diameter of the brachial artery or \%FMD (Table 1). CMV seropositivity decreased the \%FMD in RTR compared with CMV negative RTR ( $p=0.066)$, but this effect was not detected in controls.

The optimal GLM $\left(\mathrm{R}^{2}=0.317, \mathrm{p}<0.0001\right)$ included the presence of CMV lysate antibody $(p=0.068)$, age $>55$ years $(p=0.013)$, gender $(p<0.0001)$ and transplantation $(p<0.0001)$. The outcome was similar using CMV gB antibody $\left(\mathrm{R}^{2}=0.317, \mathrm{p}<0.0001\right.$ with $\mathrm{p}=0.065$ for $\left.\mathrm{gB}\right)$ but there was no link with CMV IE-1 antibody $\left(\mathrm{R}^{2}=0.317, \mathrm{p}<0.0001\right.$ with $\mathrm{p}=0.381$ for IE-1). In all models tested, BMI and blood pressure (systolic or diastolic) showed no significant association with FMD.

Measures of cIMT did not differ between RTR or controls irrespective of CMV status. There was no relationship between the presence of CMV antibodies and maximum cIMT values, with the optimal GLM $\left(\mathrm{R}^{2}=0.29, \mathrm{p}<0.001\right)$ being based entirely on age $(\mathrm{p}<0.0001$, linear term: mean cIMT increased by 0.074 per decade of life). Plaques were detected in only 11 cases, so Fisher's Exact tests were used to assess associations with the independent variables. These analyses associated 
the presence of plaques with transplantation (Fishers test; $\mathrm{p}=0.003$ ), but found no link with CMV lysate antibody $(p=0.64)$, IE-1 antibody $(p=0.72)$ or $g B$ antibody $(p=0.56)$.

Overall there was a weak association between CMV seropositivity and the earliest marker of vascular dysfunction (\%FMD), but not with cIMT, blood pressure or the presence of plaques. The negative findings may reflect the use of antibodies to mark the burden of CMV disease, the small sample size and/or the assessment of individuals with minimal or no symptoms of CMV replication or cardiovascular disease. The absence of CMV replication may be critical as there are additional mechanisms by which active CMV replication may cause vasculopathy - this includes the induction of arginase II or endothelin receptor type B $(18,19)$. Alternatively TLR2 mediates interactions between CMV and platelets that lead to pro-angiogenic responses (20) and there may be direct effects of proteins encoded by CMV, such as HCMV UL7 - a homologue of the CEACAM1 pro-angiogenic factor (21).

CMV antibody levels must be interpreted in the context of a lifetime of chronic exposure. In HIV patients, a higher CMV antibody level may mark a better immune response and consequently lower CMV replication (22). Alternatively, higher CMV antibody titres may suggest repeated reactivations of CMV antigens in individuals with subtle immune deficiencies and chronic immune activation, explaining the high titres seen in patients with advanced HIV disease or chronic obstructive pulmonary disease (23). It is also possible that these conditions (like renal transplantation) potentiate reactivations of CMV. Here CMV antibody levels rose with age and with transplantation, both of which affected FMD so the role of CMV is difficult to discern. We are now evaluating more robust measures of the burden of CMV in the context of vascular biology and transplantation.

\section{AUTHORS CONTRIBUTIONS}

PP initiated and supervised the study and prepared the manuscript, SL assessed CMV antibodies, JA recruited study subjects and tabulated the data, RP carried out statistical analyses, LN and GW managed the cardiovascular assessments and AI designed and managed aspects of the work pertaining to renal transplantation. 


\section{ACKNOWLEDGEMENTS}

The authors thank the renal transplant patients and control subjects who participated in this study, and Ms Anne Wargner, Ms Lisa Rich, Ms Nandini Makwana and Mr Alvin Borce for assistance with the recruitment and clinical assessment. The project was supported by the National Health and Medical Research Council of Australia (Grant 1068652).

\section{REFERENCES}

1. Fishman JA. Infection in solid-organ transplant recipients. N Eng J Med 2007; 357:2601-14.

2. Peterson PK, Anderson RC. Infection in renal transplant recipients. Current approaches to diagnosis, therapy, and prevention. Am J Med. 1986;81(1A):2-10.

3. Helanterä I, Schachtner T, Hinrichs C, Salmela K, Kyllönen L, Koskinen P, et al. Current characteristics and outcome of cytomegalovirus infections after kidney transplantation. Transpl Infect Dis. 2014;16:568-77.

4. Smith JM, Corey L, Bittner R, Finn LS, Healey PJ, Davis CL, McDonald RA. Subclinical viremia increases risk for chronic allograft injury in pediatric renal transplantation. J Am Soc Nephrol. 2010; 21:1579-86.

5. Chen SJ, de Craen AJ, Raz Y, Derhovanessian E, Vossen AC, Westendorp RG, et al. Cytomegalovirus seropositivity is associated with glucose regulation in the oldest old. Results from the Leiden 85-plus Study Immun Ageing. 2012; 9:18.

6. Jardine AG, Gaston RS, Fellstrom BC, Holdaas H. Prevention of cardiovascular disease in adult recipients of kidney transplants. Lancet. 2011; 378(9800):1419-27.

7. Courivaud C, Bamoulid J, Chalopin JM, Gaiffe E, Tiberghien P, Saas P, Ducloux D. Cytomegalovirus exposure and cardiovascular disease in kidney transplant recipients. J Infect Dis. 2013; 207:1569-75.

8. Izadi M, Fazel M, Saadat SH, Nasseri MH, Ghasemi M, Dabiri H, et al. Cytomegalovirus localization in atherosclerotic plaques is associated with acute coronary syndromes: report of 105 patients. Methodist Debakey Cardiovasc J. 2012; 8:42-6.

9. Gredmark-Russ S, Dzabic M, Rahbar A, Wanhainen A, Björck M, Larsson E, et al. Active cytomegalovirus infection in aortic smooth muscle cells from patients with abdominal aortic aneurysm. $\mathrm{J}$ Mol Med (Berl). 2009; 87:347-56.

10. Haarala A, Kähönen M, Lehtimäki T, Aittoniemi J, Jylhävä J, Hutri-Kähönen N, et al. Relation of high cytomegalovirus antibody titres to blood pressure and brachial artery flow-mediated dilation in young men: the Cardiovascular Risk in Young Finns Study. Clin Exp Immun. 2012; 167:309-16. 
11. Safaie N, Ghotaslou R, Montazer Ghaem H. Seroprevalence of cytomegalovirus in patients with and without coronary artery diseases at Madani Heart Center, Iran. Acta Med Iran. 2010; 48:403-6.

12. Lorenz MW, Markus HS, Bots ML, Rosvall M, Sitzer M. Prediction of clinical cardiovascular events with carotid intima-media thickness: a systematic review and meta-analysis. Circulation. 2007; 115:459-67.

13. Deanfield JE, Halcox JP, Rabelink TJ. Endothelial function and dysfunction: testing and clinical relevance. Circulation. 2007;115: 1285-1295.

14. Stein JH, Korcarz CE, Hurst RT, Lonn E, Kendall CB, Mohler ER, et al. Use of carotids ultrasound to identify subclinical vascular disease and evaluate cardiovascular disease risk: A consensus statement from the American Society of Echocardiography carotid intima-media thickness task force. J Am Soc Echocardiogr. 2008; 21:93-111.

15. Thijssen DH, Black MA, Pyke KE, Padilla J, Atkinson G, Harris RA, et al. Assessment of flowmediated dilation in humans: a methodological and physiological guideline. Am J Physiol Heart Circ Physiol, 2011; 300:H2 - 12.

16. Woodman RJ, Playford DA, Watts GF, Cheetham C, Reed C, Taylor RR, et al. Improved analysis of brachial artery ultrasound images using a novel edge-detection software system. J Appl Physiol. 2001; 91:929-37.

17. Potter K, Green D, Reed C, Woodman R, Watts G, McQuillan B, et al. Carotid intima-medial thickness measured on multiple ultrasound frames: evaluation of a DICOM-based software system. Cardiovascular Ultrasound. 2007; 5:29.

18. Yaiw KC, Mohammad AA, Costa H, Taher C, Badrnya S, Assinger A, et al. Human Cytomegalovirus up-regulates Endothelin Receptor Type B: Implication for vasculopathies? Open Forum Infect Dis. 2015; 2(4):ofv155. doi: 10.1093/ofid/ofv155.

19. Yaiw KC, Mohammad AA, Taher C, Wilhelmi V, Davoudi B, Strååt K, et al. Human cytomegalovirus induces up-regulation of arginase II: possible implications for vasculopathies. Basic Res Cardiol. 2014; 109:401. doi: 10.1007/s00395-014-0401-5.

20. Assinger A Kral JB, Yaiw KC, Schrottmaier WC, Kurzejamska E, Wang Y, et al. Human CMVplatelet interaction triggers toll-like receptor 2-dependent proinflammatory and proangiogenic responses. Arterioscler Thromb Vasc Biol. 2014; 34:801-9.

21. MacManiman JD. Human cytomegalovirus-encoded pUL7 is a novel CEACAM1-like molecule responsible for promotion of angiogenesis. MBio. 2014; 5(6):e02035.

22. Gianella S, Morris SR, Tatro E, Vargas MV, Haubrich RH, Daar ES, et al. Virologic Correlates of Anti-CMV IgG Levels in HIV-1 Infected Men. J Infect Dis. 2014; 209:452-6.

23. Tan DB, Amran FS, Teo T-H, Price P, Moodley YP. Levels of CMV-reactive antibodies correlate with the induction of CD28null $\mathrm{T}$ cells and systemic inflammation in chronic obstructive pulmonary disease (COPD). Cell Mol Immunol. 2015; doi: 10.1038/cmi.2015.04. 
Table 1: Levels of CMV antibody and FMD values are higher in RTR than control subjects.

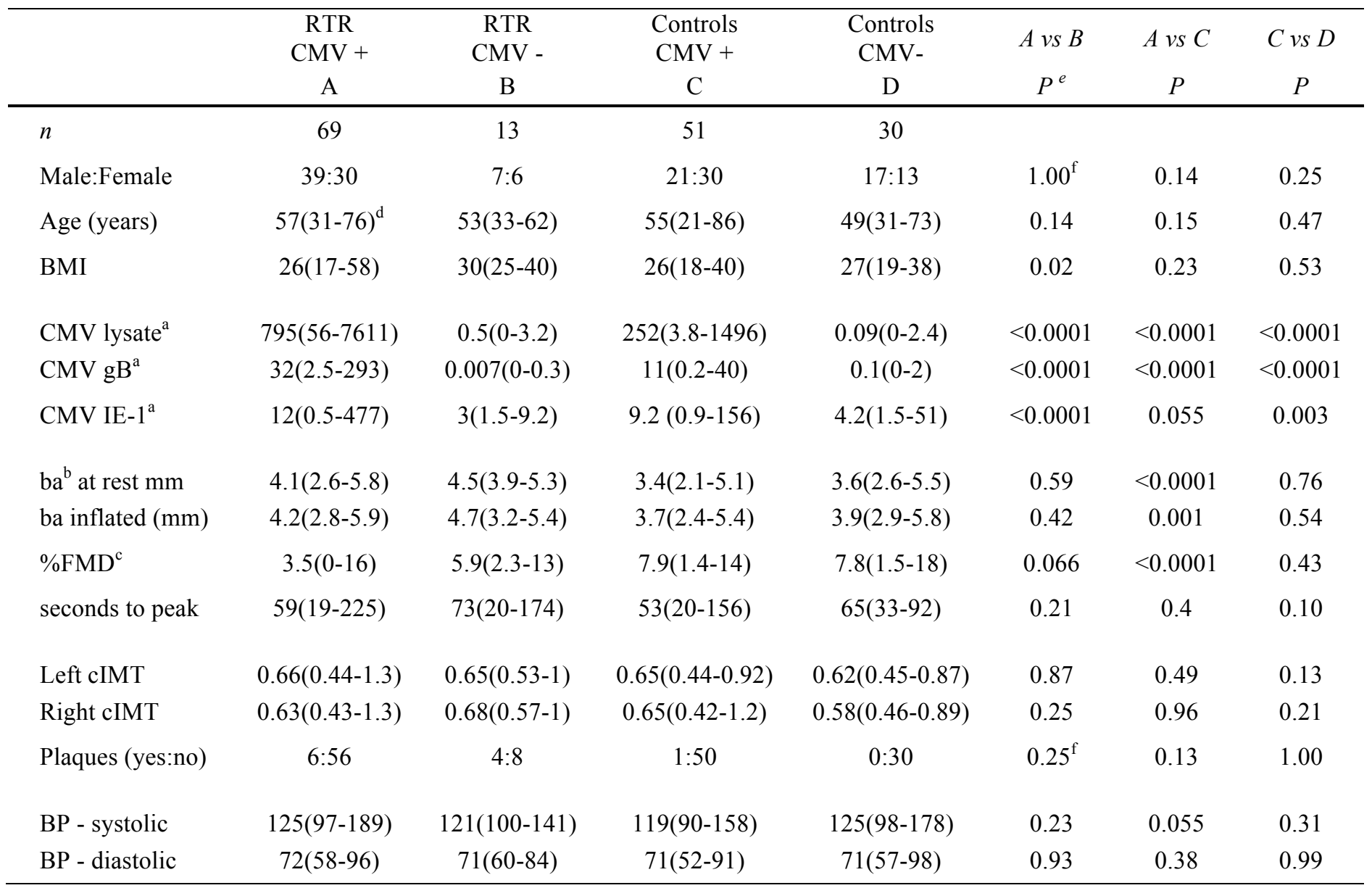

a antibody assessed in $\mathrm{AU} / \mathrm{ml}$ divided by 1000

b diameter of brachial artery (ba)

c [(ba inflated-ba at rest)/ba at rest $] \times 100$

$\mathrm{d}$ median (range) for all continuous variables

e Mann Whitney Rank Sum test unless otherwise specified

f Fisher's Exact test 